\title{
Tactical Conflict Detection with Altitude Restrictions in Terminal Airspace
}

\author{
Huabin Tang ${ }^{1}$ \\ NASA Ames Research Center, Moffett Field, CA 94035
}

\begin{abstract}
Automated conflict detection in terminal airspace is a challenging problem, in part because key information regarding flight intent is not available. Unlike en route controllers, terminal controllers currently do not enter altitude clearances when they issue them by voice, so the altitude at which a flight will level off is unavailable to the conflict detection automation. This uncertainty increases the likelihood of false alerts. Requiring terminal controllers to make entry of altitude clearances may be met with resistance due to workload concerns. A new method is studied in which a ground-based trajectory predictor predicts altitude leveloffs a priori, based on altitude restrictions on published runway descent profiles and at waypoints on Nominal Interior Routes (NIRs) and Area Navigation (RNAV) Departure Procedures (DPs). The full benefit of the intent information of all altitude clearances is shown to be achievable when controllers only make entry of altitude clearances not attributable to altitude restrictions, which leads to an insignificant workload increase; an unacceptable level of workload increase may result if all altitude clearances are entered during busy hours. In addition, the integration of altitude restriction increases the average alert lead time to 43 seconds from 37 seconds without.
\end{abstract}

\section{Introduction}

ODAY air traffic controllers are responsible for separating air traffic operating under Instrument Flight Rules (IFR). One of the primary limitations of airspace capacity is controller workload. A reliable automation tool that assists controllers in their constant estimation of potential losses of separation may help reduce controller workload, providing safer operations.

\footnotetext{
${ }^{1}$ Aerospace Engineer, M/S 210-10, Moffett Field, CA 94035, huabin.tang-1@nasa.gov.
} 
The inherent complexities of terminal operations pose a number of challenges in the development of automated tools that alert air traffic controllers to potential conflicts. Routine large-angle turns without well-defined intent information before final approaches requires proper handling to prevent a proliferation of nuisance alerts: alerts that do not provide useful information beyond what the controllers know and are not necessary to maintain safety [1]. Spacing of aircraft near standard separation thresholds to maximize arrival and departure throughput increases the difficulty of predicting separation conflicts without causing false alerts: alerts on predicted losses of separation that do not materialize, with no indication of any controller or pilot intervention [2]. The difficulty also stems from the dynamic and complex nature of the standard separation criteria, which depend on relative course, aircraft weight classes, distance to the runway, and other factors [3].

The Common Automated Radar Terminal System (CARTS) [4], which has been operational since the 1980s, and the recent Standard Terminal Automation Replacement System (STARS) [5] have a Conflict Alert (CA) functionality that generates safety alerts when CA determines that two aircraft are in dangerous proximity to one another or are on a course that will put them in dangerous proximity to one another. The Federal Aviation Administration (FAA) analyzed CA performance and classified $80 \%$ of CA alerts in terminal airspace as nuisance $[1,6]$. The analysis suggested flight-intent information to be incorporated in conflict detection algorithms so the nuisance alerts may be reduced.

A ground-based initial prototype system that incorporates flight intent information was studied in Ref. [2]. The system called Terminal Tactical Separation-Assured Flight Environment (T-TSAFE) took into account available flight-intent information to predict trajectories and employed a single-trajectory algorithm for conflict detection. It intended to alert controllers in the Terminal Radar Approach Control (TRACON) facilities to predicted losses of separation based on the separation standards with a look-ahead time of approximately two minutes. It was shown that the average alert lead time - time to first loss of separation (LOS) - was 38 seconds, based on T-TSAFE analysis of operational error cases, and the false alert rate -percentage of false alerts among the total number of alerts - was approximately $10 \%$, based on an analysis of alerts generated from the playback of real-world traffic data. In Humanin-the-Loop (HITL) experiments, controller participants using T-TSAFE provided positive feedback [7-9].

However, the operational concept for this initial prototype system required controllers to make keyboard entry of every altitude clearance, which is not the current practice in TRACON operations. Ref. [2] simulated this operational concept for incorporating level intent information with an Inferred Altitude Clearance (IAC) method, in which level 
altitudes of flights were first inferred from recorded air traffic data and then inserted back as altitude amendments. While these altitude entries provide other benefits, such as helping TRACON controllers to keep track of the altitude clearances without the need for a flight data strip [9], Subject Matter Experts (SMEs) suggest that the controllers would be reluctant or unable to make that number of entries due to the workload involved. This paper presents a new method to supplement the T-TSAFE algorithm with altitude intent information to improve conflict prediction without significantly increasing controller workload. Published altitude restrictions on waypoints and in runway altitude descent profiles are incorporated so that the controllers would only need to make entry for altitude clearances that are not attributable to altitude restrictions.

The rest of this paper is organized as follows. Section II provides details on the trajectory model based on heuristic rules and altitude restrictions. Section III explains the conflict prediction and declaration logic. Section IV describes the experiments. Section V presents the results and discussion. Section VI summarizes and concludes the work.

\section{Trajectory Model}

T-TSAFE trajectory prediction relies on aircraft current state and flight intent information, as known to the Air Traffic Control system, without reliance on numerical integration of the equations of motion. The result is the determination of an analytic kinematic trajectory, consisting of a horizontal track, a speed profile, and an altitude profile. The following subsections summarize the trajectory prediction algorithm.

\section{A. Flight Intent Information}

Flight intent information currently used by T-TSAFE includes Nominal Interior Routes (NIRs), Area Navigation (RNAV) Departure Procedures (DPs), flight plan information, and altitude clearances [2].

Flight intent information for aircraft operating in TRACON airspace is often limited due to the tactical maneuvering, called vectoring, that TRACON air traffic controllers routinely employ to separate and space aircraft in terminal airspace. While precision instrument arrival and departure procedures have been developed for many TRACONs, vectoring for spacing of arrival and departure aircraft remains common practice. Past air traffic automation efforts have attempted to predict aircraft movement in the TRACON by modeling typical flight paths that aircraft follow through terminal airspace. These are called nominal interior routes from the arrival meter fixes to the runways $[10,11]$. They are parts of the adaptation in the Time-Based Flow Management (TBFM) system [12]. Although an NIR is not a precise definition of an expected aircraft route, it helps predict aircraft trajectories by 
providing a "backbone" that aircraft typically follow with minor and transient deviations. The NIR for a flight is typically unique for given airport configuration and depends on the aircraft engine type, arrival meter fix, runway, and airport. Most arrival aircraft are observed to follow the NIRs except that a base extension or "trombone" is common for downwind approaches. Figure 1 shows a notional diagram of

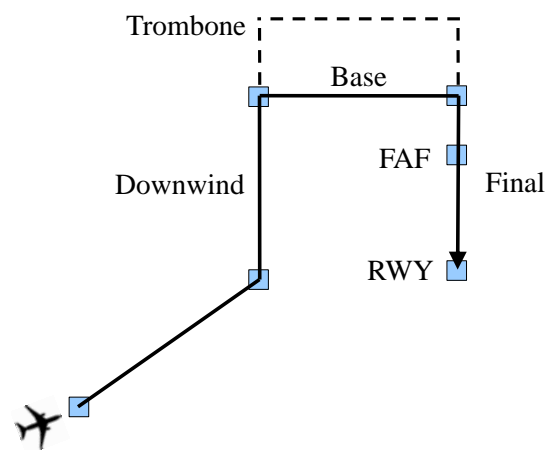
the portion of a NIR near the airport with the squares representing the waypoints including the Final Approach Fix (FAF) and runway threshold (RWY). The names of different segments of the NIR and a trombone are indicated.

Departure flights from most major airports typically follow closely the published RNAV DPs, which T-TSAFE selects dynamically based on aircraft engine type, departure meter fix, runway, and airport. Overflights are modeled to follow the filed flight plan route or, lacking a flight plan route, to progress along a dead-reckoning trajectory. The full filed flight plan for an overflight is not currently available in STARS, but a dead-reckoning trajectory would be accurate enough as there are less turns involved. STARS does have the necessary information described above for selecting NIRs and RNAV DPs. Departure runways were assumed to be given previously [2]; they are now detected automatically. Altitude clearances that are not attributable to altitude restrictions are expected to be entered into the system by controllers. However, most altitude clearances are attributable.

The Next Generation Air Transportation System (NextGen) will adopt efficient Performance-Based Navigation (PBN) arrival procedures using RNAV and Required Navigational Performance (RNP) as in the recent Terminal Spacing and Sequencing (TSAS) capability [13]. This will result in more precise flight routes with less vectoring for spacing and thus better flight intent information will become available. This would only make T-TSAFE trajectory prediction more accurate and reliable.

\section{B. Horizontal Track}

Flight trajectories in the TRACON based on NIRs were constructed in the Final Approach Spacing Tool (FAST) [11]. More recently flight trajectories based on efficient PBN arrival procedures using RNAV and RNP were constructed in TSAS [13]. Both were for the purpose of aircraft scheduling with the aircraft expected to fly the routes and, when deviating from them, heuristics were used to model its trajectory to the runway so an estimated time of arrival can be calculated. For conflict prediction on a short time horizon in the TRACON, given the current state of 
the aircraft, which can be arbitrary relative to its route, one needs to choose the most probable trajectory from many possibilities including dead reckoning with conflict probe rather than scheduling in mind. Thus, different heuristic rules for constructing the flight trajectories may be needed [2].

The horizontal track of a flight is constructed with segments of straight lines and circular arcs [14]. This is done with some heuristic rules, which depend on whether the aircraft is in conformance (on track) with its intent route -- a route defined as the merge of the NIR, RNAV DP, and flight-plan. An aircraft is on track if its cross-track distance to the centerline of its intent route is within an adjustable conformance threshold, which is chosen to be $0.5 \mathrm{nmi}$ in this work based on tests against real-world traffic data. Two basic heuristic rules are as follows [2]:

1. If an aircraft is on track, it captures the next waypoint in its intent route.

2. If it is off track, it starts with a straight line along its current course and then joins its intent route, upon interception, with circular-arc segments; otherwise it continues along a straight line.

Following the heuristic rules, one can construct the horizontal track analytically. It depends on the current position, speed, acceleration, and heading of the aircraft. An example scenario is shown in Fig. 2 in which an on-track aircraft is to fly over three ordered waypoints. In terms of the current speed $v_{0}$ of the aircraft and a bank angle $\phi_{B}$, the turn radius $r$ of the circular arc segment is approximated by

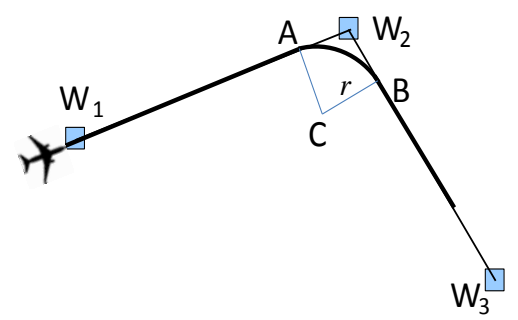

Figure 2. An on-track aircraft travels along three waypoints.

$$
r=\frac{v_{0}{ }^{2}}{g \tan \phi_{B}}
$$

where $g$ is the acceleration of gravity and a coordinated turn is assumed. The bank angle is typically chosen to be $30^{\circ}$ as most of the turns in the TRACON are large-angle turns. An aircraft at a speed of 200 knots performing a base turn along an arc of the above radius would be turning at approximately the standard rate of three degrees per second. The coordinates of the tangent points $\mathrm{A}$ and $\mathrm{B}$ and the center $\mathrm{C}$ of the circular arc, and the trajectory itself, can be determined analytically. A horizontal track consisting of segments of straight lines and circular arcs can be generated with repeated application of the general rules and this example scenario. Based on observations of a large variety of trajectories for aircraft making final approaches in real-world traffic data, additional special rules apply to aircraft near different segments of the NIR as discussed in the following. 


\section{Base Turn}

A turn to the base cannot be assumed until it is actually detected because the base segment of the nominal interior route is often extended. Figure 3 illustrates an example of an on-track flight turning onto the downwind segment of an NIR and continuing along a straight line without making a base turn: a downwind aircraft is on a dead-reckoned track until a base turn is detected. The squares are waypoints and the shaded area



Figure 3. Ground-track prediction of an ontrack aircraft turning into a downwind segment. illustrates the region of conformance as described above. A turn is defined as three consecutive radar-cycles of course changes of more than 3 degrees in the same direction (left or right). If an aircraft is turning but its heading relative to its final approach course is larger than $90^{\circ}$, it is assumed to continue turning until its course is perpendicular to the final approach course. If it is not turning and its heading is more than $80^{\circ}$ from the base, it is assumed to be deadreckoning forward, otherwise it is assumed to be approaching and eventually intercepting the final approach course.

\section{Final Approach}

A flight approaching and intercepting the final approach course is generally assumed to turn and start to intercept the final approach course at some minimum perpendicular distance $d$. This is to model flight trajectories with proper intercept angles during turn-ons to the localizer. This modeling is important in conflict detection. T-TSAFE determines this real time for any final intercept from any direction while previous research made such considerations only through adding additional segments to the routes [11,13]. Figure 4 shows a prediction of the ground track for a typical scenario of turning onto the final. Observations of actual trajectories of current-day air traffic of different TRACONs suggest that a good choice for $d$ is the smaller of $20 \%$ of the width of the base segment and $1.5 \mathrm{nmi}$. If the downwind segment does not exist so the width of the base is not defined, $d=1.5 \mathrm{nmi}$ is chosen when the angle between the heading and the final is bigger than $80^{\circ}$, otherwise $d=0.6 \mathrm{nmi}$ is chosen. This is a refinement compared with Ref. [2] where $d=2.0 \mathrm{nmi}$ was used. Circular arcs and straight lines are used to construct the intercept trajectory segments. An intercept angle $\theta=30^{\circ}$ is used when possible based on the standard

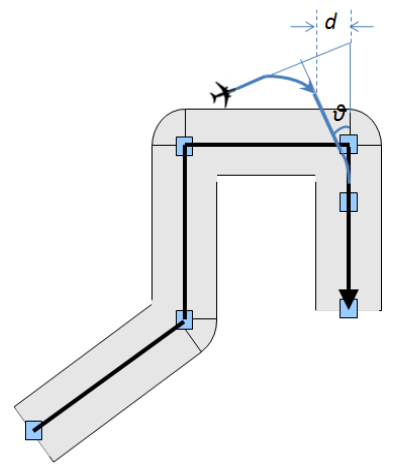

Figure 4. Prediction of the ground track of an aircraft making a base turn and a typical intercept of the final approach course. 
requirement [3]. If an aircraft is not turning and is intercepting the final approach course at $40^{\circ}$ or less, its modeled trajectory will be to fly straight forward to intercept the final. If an aircraft is already closer than $1 \mathrm{nmi}$ to the final approach course, its trajectory is assumed to turn and intercept right away. If it is not possible to turn onto the final with one circular arc, the trajectory may overshoot and intercept the final with two turns or two circular arcs [15].

\section{More Off-Nominal Cases}

Some further off-nominal situations need special attention. An off-track flight intercepting the downwind segment is modeled to turn into the segment. When it does not intercept the downwind segment, its trajectory is modeled to approach and intercept the final approach course. Thus, aircraft A and B in Fig. 5 would be modeled to turn onto the downwind segment instead of directly approaching and intercepting the final. The case of aircraft $\mathrm{A}$ is known as fanning [11]. Aircraft C and D in Fig. 6 on the other hand would have their trajectories be modeled to directly intercept the final. An aircraft approaching and intercepting the final (aircraft $\mathrm{E}$ in Fig. 5), with a heading difference of more than $130^{\circ}$ from the final approach course, and is at a position between the final approach fix and the runway and between the downwind and the final segments, is assumed to fly straight forward and does not start to turn to intercept the final until it has passed the final approach fix. A situation such as aircraft $\mathrm{F}$ is rare and it would be modeled with dead reckoning.

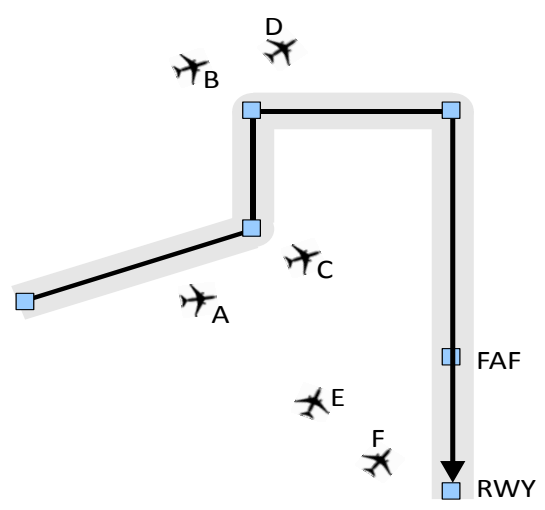

Figure 5. Various aircraft at different off-track locations relative the final approach course.

\section{Speed Profile}

An aircraft is assumed to fly along the constructed horizontal track with a kinematic speed profile. Ground speed, instead of air speed, and acceleration are used in general. This allows wind effects to be taken into account partially. To avoid reaching unrealistic speeds due to the acceleration, upper bounds for departure flights and lower bounds near the final approach and runway threshold fixes are imposed. Speed restrictions on waypoints are not currently imposed as flight intent information. Once a speed bound is reached, the aircraft is projected to fly at constant speed.

Given the current speed of an aircraft, its speed after travelling some path distance is determined, with the aircraft acceleration being the larger of its current acceleration or the one determined by the speed bound (assuming uniform acceleration). As a result, the normal speed reductions near the final approach fix and runway threshold are modeled. Once the analytic horizontal track is determined, the along-track distance between any two points on the track can be 
determined. Then the above equation allows the starting speeds at each segment of the horizontal track to be determined, as is the time duration of each segment. The aircraft position along the track as a function of time is then determined. Based on observations of real-world air traffic data, the ground speed lower bound at the final approach fix is set at 160 knots. The bounds at the runway threshold for aircraft engine types of jet, turboprop, or piston are 130,115 , or 95 knots, respectively. The ground speed upper bound for departure flights is chosen to be a relatively large value of 260 knots for flights below $6500 \mathrm{ft}$. The value of this bound is not important for most cases, and it is chosen merely to prevent the speed from becoming unrealistic in some cases of large acceleration. The ideal case would be to implement the actual speed restrictions.

\section{Altitude Profile}

The trajectory of an aircraft flying along the horizontal track, with the speed profile generated above, is complete when an altitude profile along the track is determined. The altitude profile can be modeled as one or more sub-profiles pieced together, each piece consisting of zero to two level segments and zero to one climb or descent segment. Thus, a sub-profile can be described as a climb or descent from a start altitude to a target altitude and can be modeled in three phases: an initial acceleration phase, a constant-rate phase, and a final deceleration phase [2]. If an aircraft is in the initial acceleration phase, its vertical speed at the constant-rate phase is obtained from a lookup of the nominal climb or descent rate from the Base of Aircraft Data (BADA) from Eurocontrol [16].

The level altitudes in the vertical profile are expected to come from available flight intent information. In Ref. [2], it was assumed that the controllers would make keyboard entry of all clearance altitudes. However, SMEs suggest such a requirement would encounter considerable resistance from controllers due to additional workload incurred. A new method is proposed here for obtaining the level intent information with only minor changes to current operations. There are typically well-known altitude restrictions at certain waypoints on a TRACON route (NIR or RNAV DP) or in the descent profile for a particular arrival runway. It is unnecessary to repeatedly enter the same clearance altitude for all aircraft near a particular waypoint when the altitude is attributable to the waypoint altitude restriction.

In most cases the waypoint restrictions do not depend on the route or the arrival and departure procedures. Thus, in this work, a simple approach is adopted in which the waypoint restrictions depend on some particular waypoints only. Furthermore, only altitude restrictions of "at or above" for arrival flights and "at or below" for departure flights are considered for now. Sometimes an aircraft may fly a route that does not follow precisely the nominal interior route or the route it flies may not have waypoint restrictions, so the runway descent profiles are also used, as published in 
the Instrument Approach Procedures (IAPs). The altitude restrictions are looked up in real time through tables of waypoints and runway descent profiles. Waypoint altitude restrictions often match the level altitudes in the runway descent profile; when a mismatch is found, the higher altitude restriction prevails.

Figure 6 illustrates notionally a descent profile for an arrival runway, as depicted in an IAP chart (i.e., an approach plate). The altitude restrictions include one at or above $2300 \mathrm{ft}$ at the final approach fix, $5.1 \mathrm{nmi}$ from the

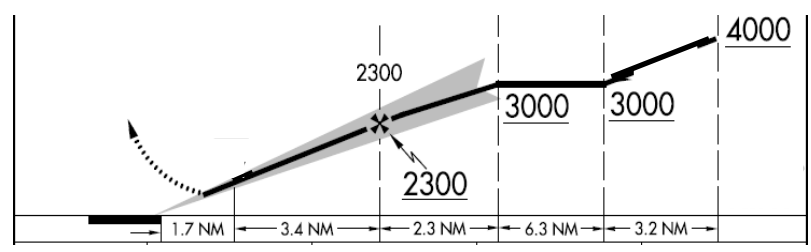
runway threshold, and others at or above $3000 \mathrm{ft}$ at 7.4 Figure 6. Illustration of a runway descent profile. $\mathrm{nmi}$ from the runway, at or above $3000 \mathrm{ft}$ at $13.7 \mathrm{nmi}$ from the runway, and at or above $4000 \mathrm{ft}$ at $16.9 \mathrm{nmi}$ from the runway. After a horizontal track is initially determined and before the speed profile is built, the trajectory is split into sub-trajectory segments based on distance to the runway threshold, and the altitude restrictions are imposed on the waypoints of the segments. A controller-assigned clearance altitude will override altitude restrictions on waypoints.

The trajectory segments with altitude restrictions on some end points together with the speed profile allow specification of the altitude restrictions at specific times. The overall altitude profile as a function of time can then be determined as superposition of sub-profiles with their start and target altitudes as well as durations calculated. Thus, the complete aircraft trajectory as a function of time needed to predict conflicts with other aircraft is determined. With a look-ahead time of two minutes, the constructed trajectory should significantly outperform dead reckoning.

\section{Conflict Prediction and Declaration}

A conflict is defined as a violation of the separation or safety requirements based on the standard in FAA Order JO 7110.65X [3]. Since the requirements in the older version of 7110.65V [17] were enforced for our air traffic data, the separation thresholds for this paper as described in Ref. [2] are based on 7110.65V. Given aircraft state information at each radar update and trajectory intent for each aircraft, computed trajectories are compared for each aircraft pair to detect instances where the applicable separation requirements are violated. Potential conflicts are detected by comparing predicted states with the required separation standards, at four-second intervals along the trajectories for the prescribed look-ahead time (two minutes). A flight-intent-based single-trajectory approach to conflict prediction is used [2] so as to reduce overall trajectory prediction uncertainty and minimize false predictions. This comes at the expense of slightly shorter lead time to a potential conflict as compared to a dual-trajectory approach [18-20]. 
When wake-turbulence separation minima are considered, the track history of the preceding aircraft may be needed to determine if the trailing aircraft is operating directly behind the leading aircraft. As a result, a track history of each aircraft for a time period at least equal to the look-ahead time must be maintained.

To minimize false alerts, we do not need to declare a conflict for every predicted violation of the separation or safety requirements. In other words, a prediction of conflict may not necessarily trigger an alert. In real-world operations, uncertainties may result from many sources such as controller vectoring, intent inference error, radar tracking noise, and trajectory modeling error. These uncertainties may lead to false alerts. After the prediction of a potential conflict, depending on how stable it is, the alerting algorithm determines if a conflict alert should be declared. The ultimate objective is to reduce false alerts while maintaining reasonable alert lead time, which is the time to first violation of the alert thresholds, defined based on the separation standards. The conventional rule of " $m$ of $n$ " cycles is adopted here, which is commonly used in Conflict Alert and elsewhere. The rule of " $m$ of $n$ " cycles in this work is in terms of the 4.8-second TRACON radar track update cycle; it means that there are $m$ predictions of violation of the alert thresholds out of $n$ radar update cycles. The $m$ and $n$ values in the " $m$ of $n$ " cycles are generally chosen based on the time to predicted first violation of the alert thresholds as shown in Table 1. Thus, any detection of the violation of the alert thresholds within 15 seconds is declared as a conflict with an alert. This is to ensure all real conflicts are detected. The longer the time to the first predicted violation of the alert thresholds, the more stringent is the " $m$ of $n$ " criterion. The numbers in Table 1 are based on observations of recent real-world traffic data and can be adjusted somewhat in practice depending on the accuracy of the track data and overall system performance.

Table 1 " $m$ of $n$ " criteria for conflict declaration

\begin{tabular}{cccccc}
\hline $\begin{array}{c}\text { Time to First } \\
\text { Conflict Prediction } \\
\boldsymbol{t} \text { (seconds) }\end{array}$ & $\boldsymbol{t} \leq \mathbf{1 5}$ & $\mathbf{1 5}<\boldsymbol{t} \leq \mathbf{3 5}$ & $\mathbf{3 5}<\boldsymbol{t \leq 6 0}$ & $\mathbf{6 0}<\boldsymbol{t} \leq \mathbf{9 0}$ & $\boldsymbol{t}>\mathbf{9 0}$ \\
\hline " $\boldsymbol{m}$ of $\boldsymbol{n}$ " criterion & 1 of 2 & 2 of 3 & 3 of 5 & 4 of 6 & 5 of 7 \\
\hline \hline
\end{tabular}

In general, a separation alert refers to one resulting from a potential violation of the required separation minima, and a safety alert refers to one resulting from a potential violation of safety alert thresholds. The former involves a pair of IFR flights, and the latter involves an IFR flight and either a Visual Flight Rules (VFR) flight or a visualapproach flight or a flight unassociated with a flight plan. A safety alert involving an IFR flight and an unassociated flight is called a Mode C Intruder (MCI) alert. When not specified, a loss of separation (LOS) in this work may refer to either a separation or a safety violation. Safety alerts involving visual-approach flights are described elsewhere [21]. 
We will distinguish between LOS and non-LOS alerts. A LOS alert means a conflict declaration that is later followed by an actual LOS, while a non-LOS alert is not followed by an actual LOS. Non-LOS alerts are further separated into valid non-LOS (VnLOS) alerts and false alerts. A false alert is generally defined as an alert to a potential LOS that is not followed by an actual LOS and for which there is no evidence of any controller or pilot intervention. A VnLOS alert is one that is not followed by an actual LOS but evidence of controller or pilot intervention exists.

The meaning of a missed alert may be ambiguous, and we will distinguish between missed prediction and missed detection of an actual LOS. A missed detection of a LOS refers to an instance in which a LOS actually occurred but the system cannot detect it even at the moment of LOS. In our approach, no missed detection is likely because when an actual LOS occurs, T-TSAFE will report it if the aircraft states are reported accurately. On the other hand, missed prediction of a LOS implies a failure to predict the LOS before some minimum lead-time threshold, e.g. 10 seconds, to allow the controller some response time.

A nuisance alert is an alert that is not useful to the controller and is thus somewhat subjective. A late (and thus nuisance) separation alert may not be useful to the controller to perform separation tasks, but it may still be useful for avoiding collisions, which is what safety alerts are for. A false alert is, by definition, a nuisance alert.

A rule adopted in T-TSAFE relevant for conflict detection and supported by SMEs is that any aircraft flying nominally level within $100 \mathrm{ft}$ of its clearance altitude is considered exactly level at its clearance altitude. In en route where aircraft altitude is generally much higher, the standard altitude rounding rule, as in the Host computer at each Center, uses a larger value of $200 \mathrm{ft}$.

\section{Experiments}

The primary objective of this work is to use published altitude intent information to effect better conflict prediction, minimizing the requirement for controllers' manual entry of altitude clearances. Two experiments were conducted to validate this. One experiment uses operational error data to show that incorporating the altitude restrictions in trajectory modeling can avoid some otherwise missed conflict predictions and may increase the average alert lead time. The other experiment uses real-world full-day air traffic data to show that application of the altitude restrictions, together with entry of altitude clearances nonattributable to altitude restrictions, may work as good as if controllers make entry of every altitude clearance. This is done by comparing T-TSAFE alerts from two methods of making level altitude intent information available: altitude-restriction (ALR) and inferred-altitude-clearance methods. In the ALR 
method, T-TSAFE deduces level altitudes attributable to altitude restrictions on the waypoints or in the runway descent profiles, without information about level altitudes nonattributable to altitude restrictions. In the IAC method, T-TSAFE simulates controller entry of all level altitude clearances, so they become available intent information, by inferring the level altitudes from analysis of the air traffic data and put them back into the data as altitude amendments.

\section{A. Experiment with Operational Error Data}

To demonstrate altitude restrictions may avoid some otherwise missed conflict predictions and increase the average alert lead time, we designed a fast-time simulation experiment that used real-world air traffic data with operational errors (failures of controllers to detect conflicts with sufficient lead time to resolve them). The average alert lead time is an indication of the effectiveness of the alerting system as it is the average time the controller has for resolving a potential LOS. While a larger alert lead time is preferable, one needs to strike a balance between average alert lead time and false-alert rate.

The experiment uses a large set of air traffic data of operational errors. Much information about the operation errors are available: standardized description of the encounters, controller-pilot communications during the conflicts, and summary of the causes and influential factors. This information helps evaluate T-TSAFE conflict predictions. The archived air traffic data were from 70 operational error cases in Dallas/Fort Worth TRACON (D10) during the period between January 2007 and April 2009. These operational errors covered a wide variety of encounters as described in Ref. [2]. Because this set of data contains all the reported actual losses of separation over a period of more than two years, it provides an unbiased set from which to measure the alert lead time.

Actual recorded air traffic data files, with TRACON radar tracking data, Mode-C barometric altitude data, and flight intent information, containing the 70 operational errors were played back through T-TSAFE first with, and then without, altitude restrictions integrated. The look-ahead time was set to 120 seconds. The output XML files of all conflict pairs were examined; alert lead times relative to the actual losses of separation were computed; and the encounters were plotted and compared with the operational-error description.

\section{B. Experiment with Full-Day Air Traffic Data}

To show that altitude restrictions may significantly reduce the need for controller altitude entries while maintaining the benefits of making entry of all altitude clearances, we designed a second experiment to compare T-TSAFE alerts from the ALR and IAC methods of obtaining level altitude intent information. Use was made of recorded full-day 
real-world traffic data of February 24, 2012 from Southern California TRACON (SCT), where CARTS was operated, and full-day data of July 26, 2014 from D10, where STARS was operated. The SCT data resulted from mixed operations that involved a mix of aircraft conducting visual and instrument approaches. The D10 data involved mostly aircraft conducting visual approaches, based on the weather conditions and the observations of aircraft making approaches with short final segments -- characteristic of visual approach procedures.

In the IAC method, before the air traffic data were played back with T-TSAFE, level altitudes of IFR flights were deduced from a pattern-matching heuristic algorithm [22] and inserted back into the data as altitude amendments. The level altitudes must last typically 20 seconds or more to be identified so false identification is reduced. The effect of altitude entries on controller workload was assessed through the number and rate of occurrence of level altitudes within the TRACON for arrivals and departures of major and non-major airports. In the ALR method, inferred altitude clearances inserted in the air traffic data were ignored. Instead T-TSAFE predicts the level altitudes attributable to altitude restrictions, although it was unable to identify the nonattributable ones.

Alerts generated under the same air traffic conditions for the ALR and IAC methods were compared. By matching the alerts pair by pair, we focus on the distinctive alert pairs that involve different aircraft pairs, rather than the common alert pairs that involve the same aircraft pairs. Specifically, we compare the number of distinctive alerts and the number of false alerts in the distinctive alerts. The results can differ between those involving major airports, for which the nominal interior routes and RNAV departure procedures are available as intent information and are closely followed by flights, and non-major airports, for which either the NIRs and RNAV DPs are unavailable or they are not followed closely at all. Thus the comparisons are made separately for major and non-major airports. Note that DAL (Dallas Love Field) will be included as a major airport because its NIRs and RNAV DPs are available and followed by flights although not as closely as those of DFW. The distinctive alerts in the ALR method may result from mismatches between the duration of the actual level-off and that modeled from the altitude restriction. The distinctive alerts in the IAC method may result from the artifact that the end of level altitudes from IAC altitude amendments are not determined until a climb or descent from that level altitude is detected.

Since it is not possible to determine whether an aircraft is on visual approach at runtime with the current data sets, all IFR arrivals are assumed to be on ILS approaches. This allows more alerts to be generated for both the ALR and IAC methods as visual-approach flights often get closer than the separation standards. Safety alerts involving visualapproach flights are thus absent, but there are MCI alerts and safety alerts involving general VFR flights. Safety alerts 
for visual-approach flights are discussed in Ref. [21]. Tracks unassociated with flight plans are inserted in the recorded air traffic data. FAA's CARTS or STARS CDR (Continuous Data Recording) data contain CA conflicts, associated and unassociated tracks, but no flight-plan information. Unassociated tracks in the CDR data were extracted and inserted into the NASA-recorded data.

The two full-day data files were played back with T-TSAFE in fast-time mode, once with the IAC method and once with the ALR method. The output XML files for conflict pairs were then analyzed. The look-ahead time was set to 90 seconds to reduce alerts from noisy radar tracks.

\section{Results and Discussion}

The results are presented in the following three subsections. Alert lead time measurements from a large number of operational error cases are analyzed in Sec. V.A with examples demonstrating the effects of altitude restrictions. TTSAFE alert rates are analyzed and compared for the inferred-altitude-clearance and altitude-restriction methods of obtaining level intent information in Sec. V.B. Specific alerts from the two methods are illustrated. In Sec. V.C, effects of altitude entry on controller workload are studied for the ALR method augmented with controller keyboard entry of altitude clearances not attributable to altitude restrictions.

\section{A. Alert Lead Time}

The alert lead times were measured from the actual first losses of separation for all conflicts in the set of 70 operational error cases. Comparison was made between the alert lead times determined for the ALR method, in which T-TSAFE dynamically extracted level-altitude intent information from altitude restrictions, and for the baseline method, in which T-TSAFE did not have any level-altitude intent information. When necessary, parameters of the trajectory model were adjusted to ensure all losses of separation were produced without missed detections.

Analysis of the XML output from fast-time playback of the traffic data files involving the operational-error cases allows us to determine the cumulative probability of alerting before any given (alert lead) time measured from first actual loss of separation. A plot of the alert cumulative distribution function of alert lead time is presented in Fig. 7 for the baseline and ALR methods. The cumulative probability for an alert lead time, $t$, measures the percentage of the 71 LOS alerts (one of the operational errors involves three aircraft in two conflicts) that have an alert lead time greater than $t$. The probability of unity at $t=0$ indicates that all 71 losses of separation were detected at or before LOS without missed detections. As can be seen, the alert lead time is improved with the ALR method. The percentage of 
alerts with lead time greater than 30 seconds for the baseline and ALR methods are $63 \%$ and $56 \%$, respectively, which are slightly less than the corresponding value of $67 \%$ in Ref. [2]. This is due to the use of stronger " $m$ of $n$ " filtering criteria for conflict declaration here. The average alert lead times calculated from the cumulative distribution functions shows an

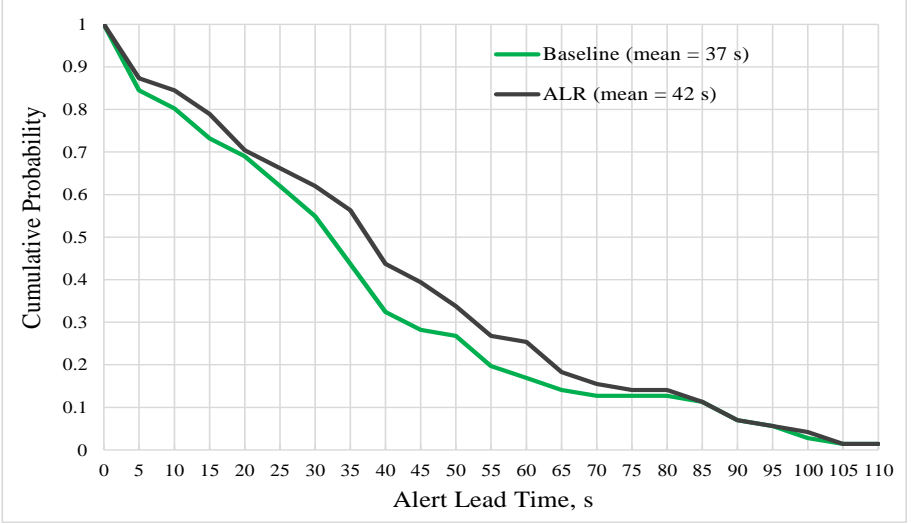

Figure 7. Alert cumulative distribution function of time for the baseline and altitude restriction methods.

increase from a baseline value of 37 seconds to 43 seconds for the ALR method. Thus, the ALR method of incorporating level intent information improves the average alert lead time by about $16 \%$ as compared to the baseline. Also, the improvement in aircraft trajectories helped increase the average alert lead time so the baseline value remains about the same as in Ref. [2] where the " $m$ of $n$ " criteria were not used, i.e. a "1 of 2" criterion was used for all predictions. Note that if we define miss prediction of LOS as those that have an alert lead time less than 10 seconds, the ALR method helps reduce miss prediction from a baseline value of $20 \%$ to $15 \%$, a $25 \%$ reduction as seen in Fig. 7. As noted in Ref. [2], the relatively large number of miss predictions result mostly from unpredictable errors that might be avoidable by trial planning of maneuvers. Study of the operational error reports shows that Conflict Alert provides negative alert lead time (to loss of separation) since it either provided no alerts or only alerted after the losses of separation. This result is expected since the safety alert criteria for CA are much smaller than the standard separation minima. The effects of altitude restrictions on the alert lead time can be demonstrated with the following two examples.

Figures 8 shows the ground tracks and altitude profiles in a case in which altitude restrictions help to increase alert lead time. This was an operational error due to the controller's "failure to project future status" of the aircraft per the standard operational error description. The two aircraft, A219 and E534, were conducting simultaneous ILS approaches to DFW runways $17 \mathrm{C}$ and $17 \mathrm{~L}$ respectively. The circles are $3 \mathrm{nmi}$ in diameter and represent the location of first LOS based on the time of first LOS determined by T-TSAFE. The stars represent time progression in oneminute intervals near the LOS. The squares represent the waypoints on the nominal interior routes. The solid line is for A219 and the dashed line for E534, with the arrows indicating the directions of flight. Note that the nominal interior routes for both aircraft only indicate the shortest final segments, and the controller has the freedom to extend the base segments through vectoring. The altitude profiles of the two aircraft are for locations near the first LOS positions; 
time origin has been set at the time of the first LOS. Aircraft A219 leveled at $6000 \mathrm{ft}$ while E534 leveled at $5000 \mathrm{ft}$. Aircraft A219 began a descent out of $6000 \mathrm{ft}$ prior to aircraft E534 being established on the runway $17 \mathrm{~L}$ localizer, leading to the LOS. If altitude restrictions in the descent profiles were not available to T-TSAFE, T-TSAFE would not have detected the LOS before the descent of aircraft A219 was detected. However, when the altitude restrictions were made available to T-TSAFE, it anticipated the descent and alerted with a lead time of 46 seconds. In contrast, the controller was unaware that an operational error was developing and neither contemplated nor took any corrective action, as stated in the operational error description.

Note that an aircraft is considered to be established on its localizer if it is within the angular course defined by the starting point at the localizer transmitter at $1000 \mathrm{ft}$ from the far end of the approach runway and expanding along the runway centerline with a full-scale course width of $700 \mathrm{ft}$ at the runway threshold [23]; the actual length of the arrival runway is used. In addition, we require the course heading of the aircraft to be within 10 degrees of the final approach course. This value was determined so that all losses of separation of the operational errors were produced.

The ground tracks and altitude profiles in Fig. 9 show a case in which the alert lead time decreased when altitude restrictions were integrated in T-TSAFE. Aircraft W516 and K229 were cleared to make simultaneous ILS approaches to runways $17 \mathrm{C}$ and $18 \mathrm{R}$ at DFW respectively. The circles, stars, squares, and arrows have the same meanings as before. The controller erroneously issued an altitude clearance to W516 to descend to $4000 \mathrm{ft}$ but intended to provide a 5000-ft altitude clearance. Meanwhile, a tricky readback error
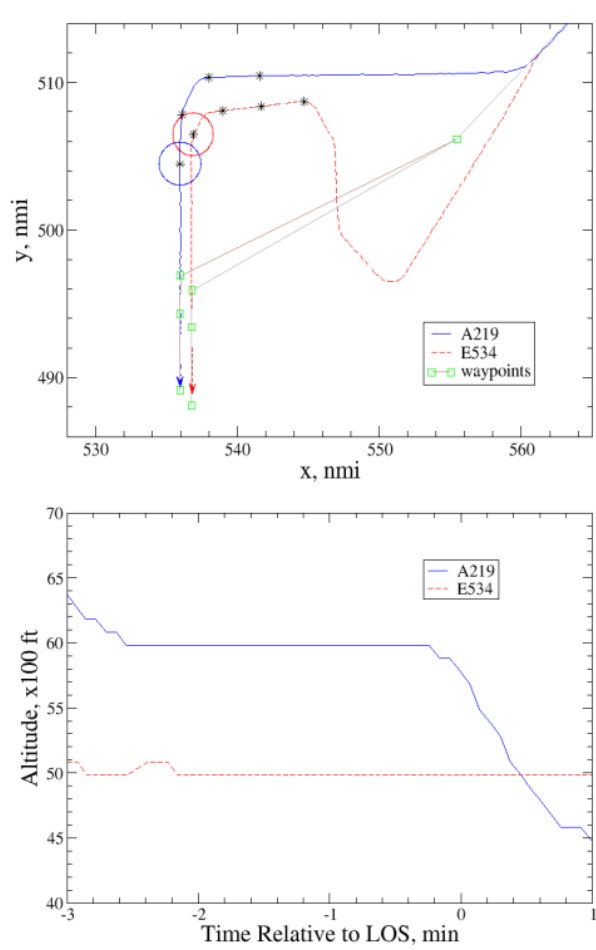

Figure 8. Ground tracks and altitude profiles of a conflict for which altitude restrictions help increase alert lead time.
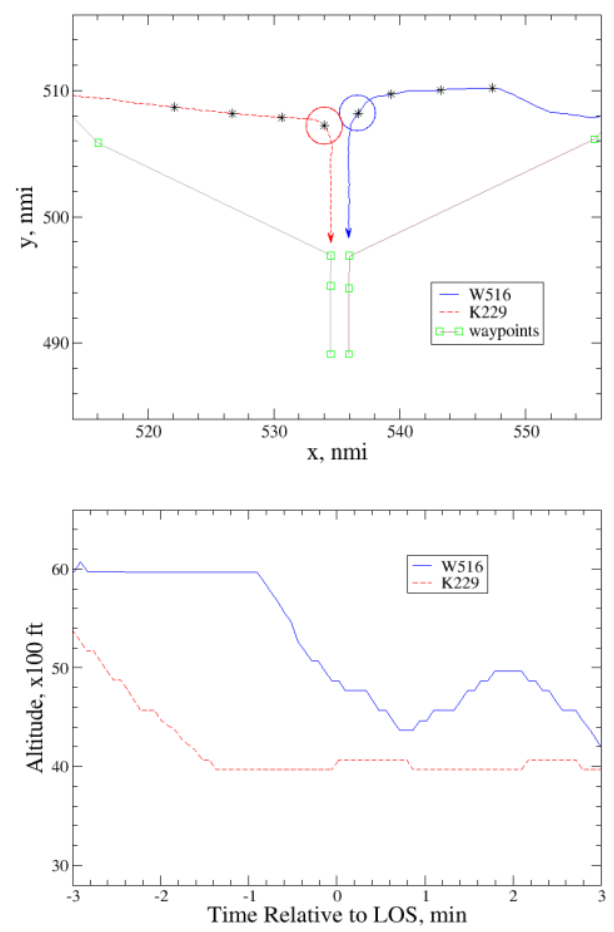

Figure 9. Ground tracks and altitude profiles for a conflict for which altitude restriction reduces alert lead time. 
occurred: W516 flight crew read back the clearance as $5000 \mathrm{ft}$ instead of $4000 \mathrm{ft}$ so the controller did not realize the error. Aircraft K229 was level at $4000 \mathrm{ft}$, so a loss of separation occurred when W516 continued to descend through $5000 \mathrm{ft}$. The controller instructed the pilot of W516 to climb back up to $5000 \mathrm{ft}$ later on. The level of $5000 \mathrm{ft}$ is an altitude restriction for runway $17 \mathrm{C}$. When altitude restrictions were integrated, T-TSAFE did not alert until the altitude level was penetrated and thus alerted with zero alert lead time. Without the level-intent information, the conflict was predicted with a lead time of 18 seconds, but that would have been a nuisance alert had the controller issued the 5000$\mathrm{ft}$ altitude clearance. Note that a dual-trajectory approach [18-20] could alert earlier though the alert would be a nuisance as well if the 5000-ft clearance were issued. The balance is in favor of a single-trajectory approach.

\section{B. Alert Rates and Analysis}

The results for the inferred-altitude-clearance and altitude-restriction methods for incorporating level altitude intent information are compared in this section based on air traffic data from Southern California TRACON and Dallas/Fort Worth TRACON. As described earlier, the ALR method uses altitude restrictions to derive the corresponding level altitudes dynamically while the IAC method emulates a concept of operation whereby controllers make manual entry for every altitude clearance.

\section{Rates of Different Types of Alerts at SCT}

To provide a baseline for comparison of the alerts from the ALR and IAC methods, we first compare alert rates at SCT for the IAC method and the baseline method in which altitude restrictions or level altitudes are not known to the system at all. The comparison is done separately for major and non-major airports.

Figure 10 shows the hourly LAX alert rates for LAX traffic, one or both aircraft are LAX arrivals or departures, in the IAC and baseline (BL) methods. The alert rates have been averaged over 24 hours. Four alert types are depicted in Figure 10: Common Loss of Separation (C-LOS) alerts represent LOS alerts that were common to both the IAC and BL methods; Common non-LOS (C-nLOS) alerts represent non-LOS alerts that were common to both methods; Distinctive IAC (D-IAC) alerts represent alert pairs that were generated by the IAC method but not by the BL method; and Distinctive BL (D-BL) alerts represent alerts that were generated by the baseline

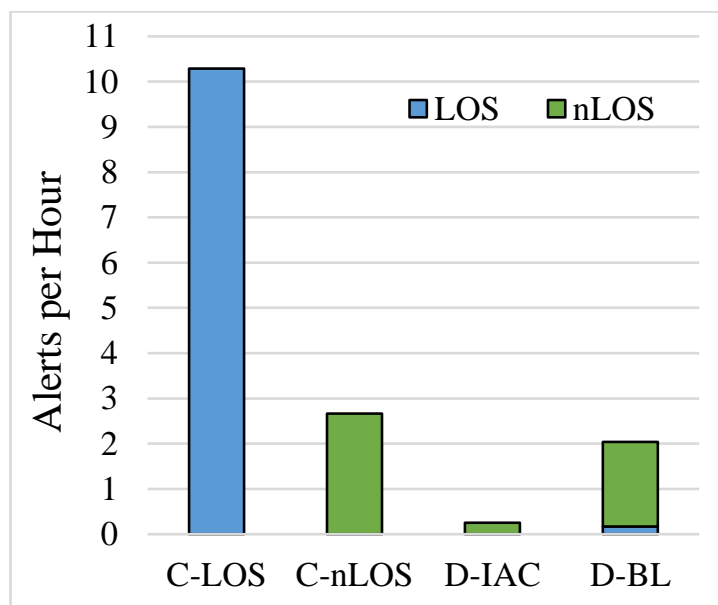

Figure 10. Rates for different types of LAX alerts in the IAC and baseline methods. 
method but not by the IAC method. As explained in Sec. IV.B, the observed weather condition and traffic pattern for the specific day suggest that arrival traffic was a mix of visual and ILS approaches, but for this simulation test, TTSAFE was configured to treat all arrivals as conducting ILS approaches so as to reduce ambiguity. So visualapproach safety alerts were not, and MCI alerts and VFR safety alerts were, included in Fig. 10. As mentioned earlier, a violation of the safety alert thresholds was included as a LOS alert in the C-LOS bar.

As seen in Fig. 10, the main difference between the IAC and baseline methods lies in the distinctive alerts represented by the D-IAC and D-BL bars, with the D-IAC nLOS alerts being only $12 \%$ of the D-BL alerts. Clearly the D-BL alerts result from lack of the level altitude intent available in the IAC method and are thus nuisance alerts, given that level altitude information will be available through altitude restrictions or altitude entries. The difference (D-BL minus D-IAC bar) between the distinctive alerts in the two methods is $40 \%$ of all non-LOS alerts in the baseline method (C-nLOS plus D-BL bar). This means that $40 \%$ of the non-LOS LAX alerts in the baseline method would not be alerted when level-altitude intent information is available through the IAC method. LOS alerts are typically common to both methods except for some rare special cases (nuisance alerts) in which the radar tracks fluctuate about an otherwise level altitude. When the fluctuations are within $100 \mathrm{ft}$, the IAC method rounds it off as if there is no fluctuation. The baseline method is unable to do so for lack of the level intent information. This may lead to LOS alerts when the fluctuations result in the loss of the vertical separation because the rule to ignore fluctuation within $100 \mathrm{ft}$ of level altitudes cannot be applied.

Alerts on non-LAX traffic were similarly studied where none of the aircraft pair involved was an LAX arrival or departure. These alerts involve overflights or aircraft arriving or departing from 29 non-major SCT airports, for which the NIRs or RNAV DPs and waypoint restrictions are either unavailable or not closely followed. The results showed that more nLOS alerts appear and the IAC method eliminates only $20 \%$ of the total nLOS alerts in the baseline method.

Similar comparisons were done between the IAC and ALR methods. Figure 11 shows the hourly alert rates for four types of LAX alerts: LOS alerts common for both ALR and IAC methods (C-LOS), non-LOS alerts common for both methods (C-nLOS), distinctive alerts in the IAC method but

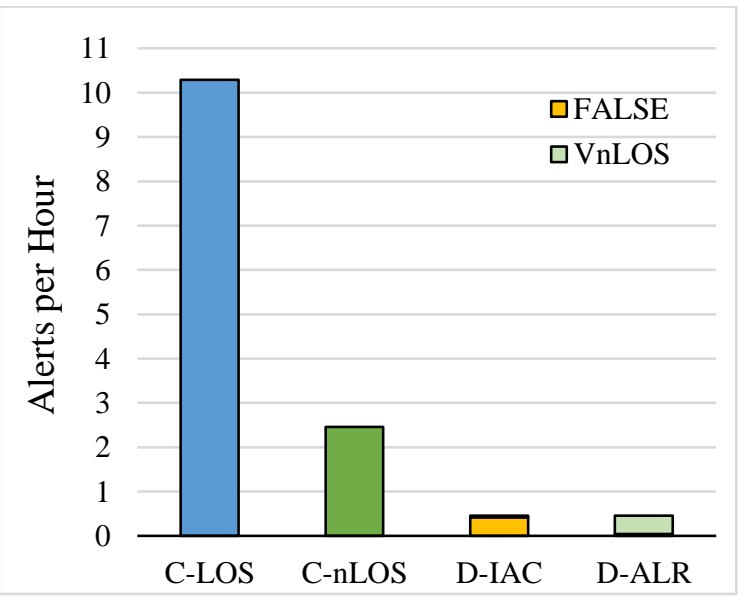

Figure 11. Rates for different types of LAX alerts in the IAC and ALR methods. 
not in the ALR method (D-IAC), and distinctive alerts in the ALR method but not in the IAC method (D-ALR). The difference in alerts between the two methods lies in the equal number of distinctive alerts represented by the D-IAC and D-ALR bars. There were a total of 11 alerts in the D-IAC bar, 10 of which were false and only one was valid. All 11 alerts in the D-ALR bar were valid with 10 VnLOS alerts and one LOS alert. The distinctive alerts for each method was $16 \%$ of the total VnLOS alerts. Examples of these distinctive LAX alerts are discussed in the next subsection.

A similar comparison of LAX alerts from the ALR and baseline methods showed that the ALR method eliminated $36 \%$ of all non-LOS alerts created by the baseline method. This is comparable to the $40 \%$ reduction for the IAC versus baseline method in Fig. 10. Study of the non-LAX alert rates for the IAC and ALR methods was done as well. These alerts involve aircraft arriving or departing from 29 non-major airports in SCT. The results showed that distinctive alerts was $24 \%$ of the VnLOS alerts for the IAC method while the same ratio for the ALR method was $38 \%$.

\section{Comparison of Distinctive LAX Alerts}

To better understand different alerts from the IAC and ALR methods, three examples of LAX alerts from the distinctive alerts in the two methods are examined in this subsection. The first example is a false alert out of the 10 similar false alerts among the 11 distinctive alerts from the IAC method. The second and third examples demonstrate the distinctive VnLOS alerts from the ALR method.

Fig. 12 shows the ground tracks and altitude profiles of two aircraft involved in a false alert that was distinctive to the IAC method. Aircraft A560 is an LAX arrival turning downwind to approach runway 24R while aircraft C511 is a VNY (Van Nuys airport) arrival on a crossing track. The symbols have the same meaning as before with the circles being $3 \mathrm{nmi}$ in diameter. The light solid and dashed lines are the actual trajectories and the centers of the light circles represent horizontal Closest Point of Approach (CPA). Each dark solid circle indicates the first predicted loss of separation centered at the end of the dark solid line -- the predicted trajectory starting from the aircraft's current position. The aircraft clearly lost horizontal separation. As seen in the corresponding altitude profiles, aircraft C511 leveled off at
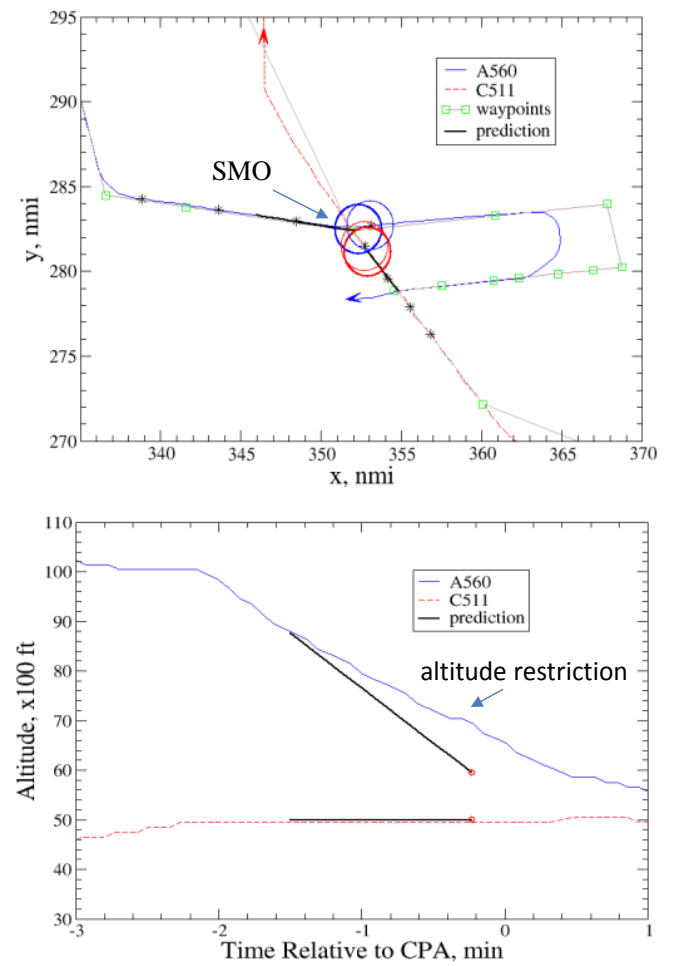

Figure 12. Ground tracks and altitude profiles of aircraft involved in a false D-IAC alert in the IAC method. 
$5000 \mathrm{ft}$ while aircraft A560 was descending out of $10000 \mathrm{ft}$. The dark solid straight lines represent the altitude profiles predicted by T-TSAFE with the end small circles indicating the altitudes at the predicted first LOS. The time is measured from the CPA. The IAC method uses the current descent rate to project the descent of aircraft A560, but A560 reduced its descent rate likely in anticipation of the (short-duration) altitude restriction at or above $7000 \mathrm{ft}$ at waypoint SMO. As a result, the IAC method falsely predicted a potential LOS to occur in about 60 seconds near SMO, which never materialized as the prediction stopped in a couple of radar update cycles when the descent rate of A560 was reduced. On the other hand, the ALR method anticipated the altitude restriction and predicts a level-off at 7000 ft near SMO, so no alert was ever generated. While there appears to be a short level-off in the actual altitude profile, it was too short to be recognizable as such by T-TSAFE with the IAC method.

The second example involves a VnLOS alert for the ALR method that was distinctive with the altitude profile of one aircraft containing a level-off that was not attributable to an altitude restriction. The ground tracks and altitude profiles of this example are shown in Fig. 13. Aircraft S450 was turning from the base onto its final to LAX runway 25L while S561 was already established on its final to LAX runway 24R. This was for an alert in the D-ALR bar in Fig. 11. The symbols mean the same as before. The circles are $3 \mathrm{nmi}$ in diameter with the light ones at the CPA and the dark ones at the predicted LOS points. The dark lines are predicted horizontal tracks. The base segment for S450 started from the indicated waypoint SLI, which has an altitude restriction at or above $7000 \mathrm{ft}$. The end waypoint of the base segment has an altitude restriction at or above $5000 \mathrm{ft}$.

Without any further intent information, we assume aircraft follow their runway descent profiles even when they are still on the base segment (not on the final approach yet). As a result, altitude restriction on the runway descent profile also requires S450 to stay at or above $5000 \mathrm{ft}$ before reaching the final segment. In practice S450 may stay level (7000 $\mathrm{ft}$ in this case) beyond the starting waypoint of the base segment without requiring the controller's further instruction. However, we assume that aircraft would start to climb or descend as soon as they have passed through a waypoint
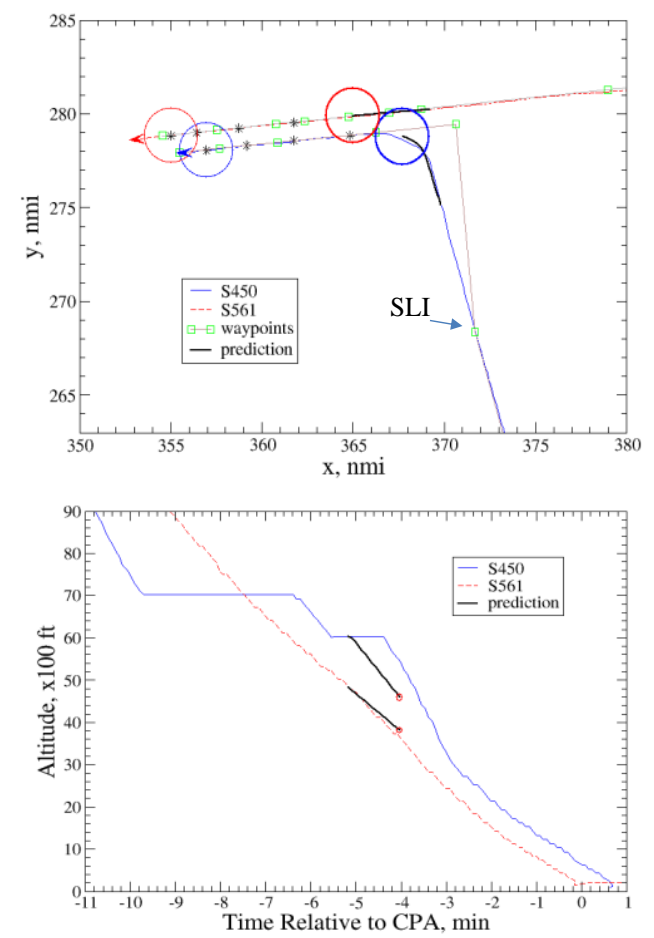

Figure 13. Ground tracks and altitude profiles of aircraft involved in a VnLOS DALR alert with a nonattributable level-off. 
of an altitude restriction, i.e. as soon as the restriction is off. This assumption is generally justified based on observations. For example, with the current traffic data, we observed that, among a large number of daily flights through all routes with this base segment, only two aircraft involved in alerts related to extension of the level-off at $7000 \mathrm{ft}$. In the present case, S450 did not begin its descent out of $7000 \mathrm{ft}$ until about $2 \mathrm{nmi}$ beyond SLI. Furthermore, as can be seen, S450 made an additional level-off at $6000 \mathrm{ft}$ nonattributable to an altitude restriction. Unaware of this level-off leads to a non-LOS alert from the ALR method. Since we expect that the ALR method should be augmented with altitude entry of nonattributable altitude clearances, which would eliminate the alert, we consider the level-off as a controller or pilot intervention and categorize this as a VnLOS alert.

The third example involves another VnLOS alert that was distinctive of the ALR method. Figure 14 shows the ground tracks and altitude profiles of aircraft D236 and S553. Aircraft D236 made an unusual vectoring off its base segment before approaching its final on LAX runway 25L while S553 was established on its final on LAX runway 24R. This is for an alert in the D-ALR bar in Fig. 11. The symbols again mean the same as before. Aircraft D236 is being vectored unexpectedly by the controller for spacing prior to merging onto the final approach. As previously discussed, vectoring is currently treated as uncertainty in the intent information in the NIR. For lack of this vectoring intent information, the predicted horizontal trajectory deviates from the actual trajectory significantly. However, this was an unusual vectoring in that it appeared late and abrupt as D236 already turned onto the base and were too close to the final while the angle of turning away from the base was more than 90 degrees. As in the case of Fig.13, there is an altitude restriction of $7000 \mathrm{ft}$ at waypoint SLI before the next restriction at $5000 \mathrm{ft}$ on the runway descent profile. The ALR method projects that D236 will descend right away after passing SLI, however, as seen in the altitude plot, it maintains 7000 $\mathrm{ft}$ for 30 more seconds while performing the vectoring until proper spacing is achieved. Thus a non-LOS alert results. This is considered a valid alert since the alert occurs right near the beginning of the unusual vectoring that should qualify as a controller intervention. This alert would help the controller be aware of the situation and take
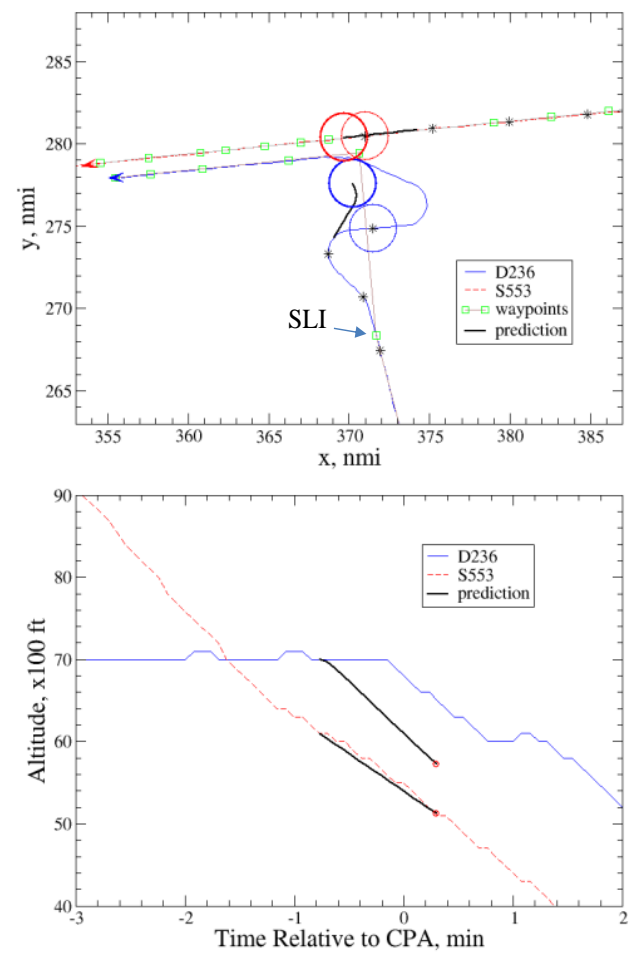

Figure 14. Ground tracks and altitude profiles for aircraft involved in a D-ALR alert with unusual vectoring. 
proper action such as not to descend until the alert is cleared. The controller could make an altitude entry of $7000 \mathrm{ft}$ to remove this alert.

Overall, within the 10 valid non-LOS alerts in the D-ALR bar in Fig.11, there were a total of five alerts similar to the second example above that involved nonattributable altitude clearances. These would not be alerted if the controllers entered nonattributable altitude clearances. There were also a total of three alerts similar to the third example, where an aircraft continue to remain level-off beyond the waypoint of altitude restriction. In fact, aircraft D236 in the third example involved in another VnLOS alert with a different aircraft on runway 24R. The other alert involved another pair of aircraft in similar setup, where the alert was triggered because the aircraft approaching runway 25L from waypoint SLI maintained $7000 \mathrm{ft}$ until right before it turned onto the localizer in order to achieve proper spacing with an aircraft established on runway $25 \mathrm{~L}$. For a small number of such cases of clearances before proper spacing is achieved, the controller should enter the clearance altitudes after the waypoint restriction is off. Note that the D-ALR alerts did not appear when the IAC was used since the aircraft would remain on the inferred altitude clearances until a descent is detected. The two other VnLOS alerts out of the 10 VnLOS D-ALR alerts involved speed or vectoring that made the alert not materialized while the altitude profiles were predicted accurate enough. So they still qualify as VnLOS and they would be the alerts that would not disappear even after additional entries of nonattributable altitudes were made.

The false alerts in the distinctive alerts from the IAC method mainly result from short-duration level altitudes at altitude restrictions that are not identifiable as IAC altitudes, as shown in the first example. This is the artifact in the IAC method for simulating the effect of controller entry of level altitudes. The ALR method of obtaining level altitude intent information through altitude restrictions avoids such drawbacks. If additional entries of nonattributable altitudes are made, most of the distinctive VnLOS alerts from the ALR method would not be issued by T-TSAFE. Thus, we conclude that the ALR method augmented with entries of nonattributable altitudes may work as well as when entries of all level altitudes are made.

\section{Analyses of Alerts at D10 TRACON}

The same analyses were done for the experiment that used the D10 traffic data. Figure 15 shows the hourly alert rates with at least one of the aircraft involved being a DFW or DAL arrival or departure. The alert rates have again been averaged over 24 hours, and the bars indicate the rate of occurrence of C-LOS, C-nLOS, D-IAC, and D-ALR alerts, respectively. As mentioned earlier, the weather conditions and the traffic pattern for the specific day suggested 
mostly visual approaches but all arrivals were assumed to be on ILS approaches instead so the standard separation criteria could be used. This allowed more alerts to be compared between the ALR and IAC methods.

As expected, the differences between alerts in the two methods lie in the distinctive D-IAC and D-ALR bars. As in the case for the SCT data, the alert rates for D-IAC and DALR are almost the same and are $21 \%$ and $23 \%$ of the total nLOS alerts for the IAC and ALR methods respectively. However the D-IAC alerts are again almost all false alerts for the same reason as the LAX alerts; that is, the intended leveloffs were too short to be recognizable. Many of those false alerts resulted from the fact that some departure flights did not appear to conform to the published $10000-\mathrm{ft}$ altitude

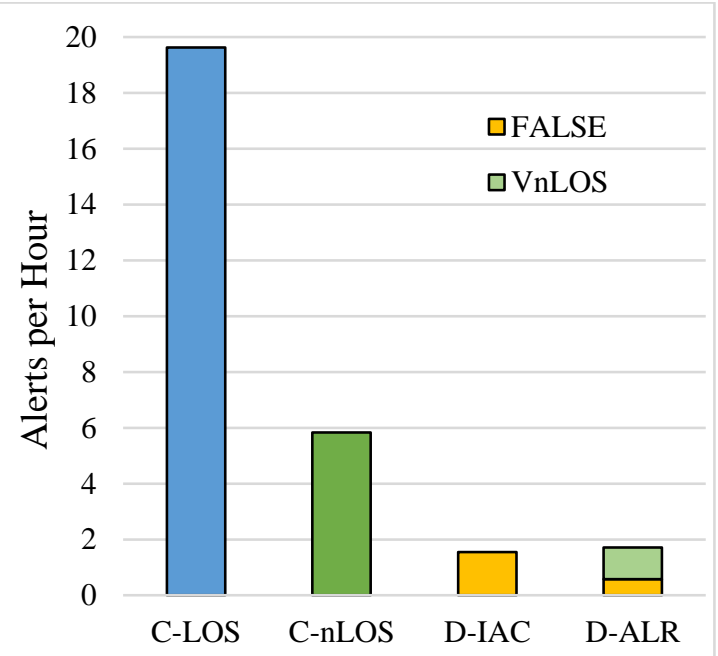

Figure 15. Rates for different types of DFW and DAL alerts in the IAC and ALR methods. restriction; instead they climbed slowly through that altitude, such that no level-off is identifiable. As a result, alerts that were not generated in the ALR method were generated in the IAC method.

The D-ALR bar contains 27 valid non-LOS alerts and 12 false alerts. The 21 out of the 27 VnLOS alerts resulted from level-offs nonattributable to altitude restrictions; the other six alerts were precluded from false alerts due to speed maneuvers or vectoring. Alerts due to unknown level-offs nonattributable to altitude restrictions would disappear when the ALR method is augmented with controller entries of the corresponding altitude clearances. Sixteen out of those 21 VnLOS alerts were DFW arrivals; the remaining five consisted of two DAL arrivals and three DFW or DAL departures. Many of the 12 false alerts lasted about 10 seconds and correspond to the level altitudes extend slightly (say 10 seconds) beyond the altitude restriction waypoint. As in the analysis based on the SCT data, one can conclude that the ALR method combined with controller entries of altitude clearances nonattributable to altitude restrictions may effectively work as well as when entries of all level altitudes are made.

The same analysis was done for the non-DFW and non-DAL alerts in which none of the aircraft involved was a DFW or DAL arrival or departure. The observed alert rates were much smaller: $0.8,0.4,0.1$, and 0.3 per hour for CLOS, C-nLOS, D-IAC, and D-ALR alerts, respectively. This is because the alerts involved aircraft arriving or departing from only eight non-major airports in D10, as compared to 29 in SCT. Thus, non-major airports for D10 do not play a significant role. 


\section{Altitude Entry and Controller Workload}

While no supporting analysis was found in the literature, SMEs suggest that workload could be too high if TRACON controllers were required to make entry of all altitude clearances. This subsection presents a first analysis in this direction.

Table 2 shows the number of level altitudes attributable and nonattributable to altitude restrictions during the busy hours from 8:00 AM to 9:00 PM local time for the SCT data on February 24, 2012. The level altitudes are categorized in terms of LAX arrivals, LAX departures, and non-LAX arrivals or departures. While 39\% of level altitudes for LAX arrivals are nonattributable, $90 \%$ of level altitudes for non-LAX flights are nonattributable due to the lack of, or aircraft not following closely, the NIRs and RNAV DPs. As seen earlier, a level-off attributable to altitude restriction can be too short to be recognized by the IAC method while a nonattributable level-off should not be too short to be recognized as it results from a specific clearance that should not be penetrated until another clearance is issued. Thus the number of attributable level-offs for LAX arrivals may well be underestimated. The level-offs nonattributable to altitude restrictions were unavailable in the ALR method by definition, however only eight distinctive alerts in the ALR method correspond to nonattributable level altitudes. Thus less than $3 \%$ of the nonattributable level altitudes of LAX arrivals led to alerts.

Table 2 Number of level altitudes attributable and nonattributable to altitude restrictions above $950 \mathrm{ft}$ for SCT during 13 busy hours.

\begin{tabular}{lccc}
\hline \hline & Attributable & Nonattributable & Total \\
\hline \hline LAX Arrivals & 550 & 354 & 904 \\
\hline LAX Departures & 72 & 287 & 359 \\
\hline Other Flights & 240 & 2172 & 2412 \\
\hline \hline
\end{tabular}

If controllers made entry of all nonattributable LAX-arrival level altitudes in Table 2, it would amount to only seven entries per hour per controller position, averaging over 13 work hours and four arrival controller positions at LAX: two feeder sectors of East Feeder and Zuma and two approach sectors of Downe and Stadium. By slicing the time into 15 minutes intervals, we extracted the number of level-offs as a function of time for each controller position, approximately defined based on distance to LAX and arrival runways. The number of level-offs at peak traffic hours can then be determined. We found only three isolated bursts of eight or nine level-offs within 15 minutes for the fullday traffic: one for each of the three controller positions of Stadium, Downe, or East Feeder. The rest were mostly 
between zero and four level-offs in 15 minutes. Thus, making entry of nonattributable level-offs does not significantly increase controller workload for LAX arrivals.

The same conclusion may not apply for attributable level-offs as they could be well underestimated. Consider the straight-in arrivals of parallel runways $24 \mathrm{R}$ and $25 \mathrm{~L}$ at LAX with the single approach controller position at Downe, there are seven and eight altitude restrictions for runways $24 \mathrm{R}$ and $25 \mathrm{~L}$ respectively in the runway descent profiles between $2000 \mathrm{ft}$ and $10000 \mathrm{ft}$, as seen from the IAP charts. In busy hours on a typical day with simultaneous parallel approaches to the two runways, we find a typical throughput to be 60 aircraft per hour. Thus, the Downe controller would need to make eight altitude entries per minute if all level-offs attributable to altitude restrictions are required to be entered. Since controllers are busy merging and separating aircraft, requiring them to make an additional 500 altitude entries per hour could be excessive. The altitude restriction method of obtaining level intent information augmented with controller entry of nonattributable altitude clearances may resolve this issue.

Table 2 suggests that the altitude restriction method may work with LAX departures as well. For non-LAX arrivals and departures, controllers may enter the level altitudes either when they are not too busy or they could wait until alerts are being generated.

The same conclusion can be drawn from similar analysis on the DFW data. The difference is that the number of inferred altitude clearances for airports other than DFW and DAL was much smaller -- only 468 within 13 busy traffic hours. A result consistent with the fact that D10 has only about one fourth as many non-major airports as does SCT.

\section{Summary and Conclusions}

In this paper, algorithms for trajectory generation and tactical conflict prediction and declaration have been reviewed and enhanced. Altitude restrictions from runway descent profiles and waypoints on the Nominal Interior Routes (NIRs) and Area Navigation (RNAV) Departure Procedures (DPs) were incorporated as intent information in the altitude profiles. Parameters were adjusted with real-world air traffic data from operational-error cases and two large TRACON facilities. Alert lead time, alert rates, and the effect of controller altitude entries on controller workload were studied.

Experiments were performed through playback of recorded real-world air traffic data. With real-world operational error data, the altitude-restriction method of gaining level altitude intent information showed an improvement of average alert lead time from 37 seconds to 43 seconds without and with altitude restrictions integrated, respectively. 
With full-day air traffic data from two TRACONs, it was shown that, for major airports where NIRs and RNAV DPs are available and are followed closely, the altitude restriction method, augmented with controller entries of only those altitude clearances not attributable to altitude restrictions, would work as well as if all altitude clearances were entered into the system by controllers. This was done by analyzing the differences between alerts in the altitude restriction method and the previous Inferred Altitude Clearance (IAC) method. The distinctive alerts -- different aircraft pairs for the two methods -- in the IAC method were mostly false; they resulted from artifacts of the method itself and would not have occurred if the controller had entered the relevant level altitudes, which were attributable to altitude restrictions. On the other hand, the distinctive alerts in the altitude restriction method were mainly due to level altitudes not attributable to altitude restrictions; they would not have occurred if the altitude clearances were entered. It was shown that the workload increase is insignificant in the altitude restriction method, enhanced with controller entries of altitude clearances not attributable to altitude restrictions for major airports. For non-major airports where the NIRs and RNAV DPs are not followed closely or not available at all, the controllers might make the entries after alerts are triggered or when they are not busy.

\section{References}

[1] Friedman-Berg F, Allendoerfer K., and Pai, S., "Nuisance Alerts in Operational ATC Environments: Classification and Frequencies", Proceedings of the human factors and ergonomics society, 2008.

[2] Tang, H., Robinson, III, J.E., and Denery, D.G., "Tactical Conflict Detection in Terminal Airspace”, AIAA, Journal of Guidance, Control, and Dynamics, Vol.34, No.2, 2011, pp 403-413.

[3] “Air Traffic Control”, Federal Aviation Administration, Order JO 7110.65X, Washington, D.C., Oct. 2017. https://www.faa.gov/documentLibrary/media/Order/JO_7110.65X_Air_Traffic_Control.pdf [retrieved 16 Nov. 2017].

[4] "Common ARTS Computer Program Functional Specifications (CPFS), Conflict Alert", Federal Aviation Administration, NAS-MD-632, Washington, D.C., Apr. 2007.

[5] "Standard Terminal Automation Replacement System (STARS), Adaptation Data Maintenance Manual, Revision 20", Raytheon, Prepared for Federal Aviation Administration, NAS-MD-4428, Washington, D.C., Jan. 2014.

[6] Allendoerfer, K., Friedman-Berg, F., and Pai, S., "Human factors analysis of safety alerts in air traffic control", (DOT/FAA/TC-07/22), 2007. 
[7] Verma, S., Tang, H., Kozon, T., Ballinger, D., and Farrahi, A.,"Initial Human-In-The-Loop Evaluation of a Tactical Conflict Detection Tool in the Terminal Area", 4th International Conference on Applied Human Factors and Ergonomics, San Francisco, July 2012.

[8] Kozon, T., Verma, S., Farrahi, A., Tang, H., and Ballinger, D., "Phase-2 Evaluation of a Tactical Conflict Detection Tool in Terminal Area", 31st Digital Avionics System Conference, Williamsburg, VA, October, 2012.

[9] Verma, S., Tang, H., Ballinger D., Chinn, F., Kozon, T., Farrahi, A., Buchmann, E., Walker, J., Wooten, D., Pfeiffer, J., Carpenter, D., and Lehmer R., "Human Factors Evaluation of Conflict Detection Tool for Terminal Area", $10^{\text {th }}$ USA/Europe Air Traffic Management Research and Development Seminar, Chicago, Illinois, June, 2013.

[10] Swenson, H. N., Hoang, T., Engelland, S., Vincent, D., Sanders, T., Sanford, B., and Heere, K., "Design and Operational Evaluation of the Traffic Management Advisor at the Fort Worth Air Route Traffic Control Center," 1st USA/Europe Air Traffic Management R\&D Seminar, Saclay, France, Federal Aviation Admin. And EUROCONTROL, Paper 012, June 1997.

[11] Robinson, III, J.E. and Isaacson, D.R., "A Concurrent Sequencing and Deconfliction Algorithm for Terminal Area Air Traffic Control”, AIAA Paper 2000-4473, Sept. 2000.

[12] “Time Based Flow Management”, Federal Aviation Administration, Washington, D.C., 2016.

https://www.faa.gov/nextgen/update/progress_and_plans/decision_support_systems/\#tbfm [retrieved 16 Nov. 2017].

[13] Robinson, J.E., Thipphavong, J., and Johnson, W.C., “Enabling Performance-Based Navigation Arrivals:

Development and Simulation Testing of the Terminal Sequencing and Spacing System", 11th USA/Europe Air Traffic Management Research and Development Seminar, Lisbon, Portugal, June, 2015.

[14] Erzberger, H. and Pecsvaradi, T., "4-D Guidance System Design with Application to STOL Air Traffic Control”, 13th Joint Automatic Control Conference, Stanford, California, August 1972.

[15] Erzberger, H. and Lee, H.Q., "Terminal-Area Guidance Algorithms for Automated Air-Traffic Control”, NASA/TN-D-6773, April 1972.

[16] "User Manual for the Base of Aircraft Data (BADA)", Rev. 3.6, Eurocontrol, Brussels, 4 July 2004.

[17] "Air Traffic Control”, Federal Aviation Administration, Order JO 7110.65V, Washington, D.C., Apr. 2014. http://www.faa.gov/documentLibrary/media/Order/JO_7110.65V.pdf [retrieved 1 Sep. 2014].

[18] Paielli, R.A. and Erzberger, H., "Tactical Conflict Detection Methods for Reducing Operational Errors", Air Traffic Control Quarterly, Vol. 13(1), 2005, pp 83-106. 
[19] Paielli, R.A. and Erzberger, H., “Analysis of False-Alert Rates for a Tactical Conflict Detection System”, AIAA Paper 2005-7473, Sept. 2005.

[20] Paielli, R.A., Erzberger, H., Chiu, and Heere, K.R., "Tactical Conflict Alerting Aid for Air Traffic Controllers", AIAA, Journal of Guidance, Control, and Dynamics, Vol.32, No.1, 2009, pp 184-193. doi: 10.2514/1.36449

[21] Tang, H., "A Tactical Separation and Safety Alerting System for Terminal Airspace", to be submitted to Journal of Air Transportation.

[22] Robinson, III, J. E., and Kamgarpour, M., "Benefits of Continuous Descent Operations in High-Density Terminal Airspace Under Scheduling Constraints," 10th AIAA Aviation Technology, Integration, and Operations (ATIO) Conference, Fort Worth, TX, 13-15 Sep. 2010. doi: 10.2514/6.2010-9115

[23] “Aeronautical Information Manual”, Federal Aviation Administration, Washington, D.C., Oct. 2017. http://www.faa.gov/air_traffic/publications/media/AIM_Basic_dtd_10-12-17.pdf [retrieved 16 Nov. 2017]. 